\title{
Design and Implementation of the Thesis Evaluation System Based on Web Technology
}

\author{
Li Bo \\ Jilin Agricultural University, Graduate School, Changchun130118, China \\ 83574736@qq.com
}

Keywords: Web technology, Java language, thesis evaluation system

\begin{abstract}
This paper introduces the research of Thesis Evaluation System Based on Web technology, the system makes Web page by HTML/CSS/JS, AJAX/JQuery, applying Struts2 framework to build the system framework, using Java language to make Oracle database as data storage and achieves the process of upload - Delivery - review.
\end{abstract}

\section{Introduction}

"Thesis" as a research student culture quality of the landmark achievements, has received more and more extensive attention from all sectors of society, colleges and universities and scientific research institutions generally think the review is to ensure that the quality of one of the key links. However, currently most of the colleges and universities use mailing the assessment method and the traditional way exists many drawbacks, in the review process is time-consuming and laborious, inefficient, has been difficult to adapt to at present demand and development of the marking of the graduate, degree of the network evaluation system development and implementation is imperative.

At the beginning of this system, we have studied the development and application of network system review papers by consulting the data, field visits, exchange visits and other forms. Through the investigation found that at present only a small part of the colleges and universities developed in the dissertation remote evaluation system, the system has been basically covered the network to submit papers, review papers, delivery of the paper, view marking the opinions of all process and the basic functions. On the scope of Jilin Province, Jilin Agricultural University is an early independent development of the use of network evaluation system, in the completion of the basic functions, to better strengthen the system compatibility and the concept of human nature.

\section{System Development Related Technologies}

Java Language. Java is one of the most widely used network programming languages. It has the characteristics of simple, object-oriented, stable, and platform independent, explanatory, multi thread, dynamic and so on.

Platform Independence. Java introduced virtual machine principle, and run on the virtual machine, built on the hardware and operating systems, data types and machine independent, can run on different platforms.

Security. When the program runs, the memory is allocated by the operating system, so that the virus can be avoided through the pointer intrusion system. Java provides security manager for the program to prevent unauthorized access to the program.

Object-oriented. The data package in the class, the advantages of the use of class to achieve the simplicity of the program and easy maintenance.

Oracle Database. Oracle database is a relational database management system, is one of the mainstream database, it has the advantages of strong availability, scalability, strong data security, stability and so on.

Strus2 Framework. Strus2 framework is currently widely used framework of. It adopts MVC architecture pattern, in the MVC pattern, the application is divided into three parts: model, view and controller, the only focus on their own business logic, each task processing, greatly improving the application flexibility and reusability. Among them, Model is used to process the application data 
logic in the application; View is the part of the application process data display; Controller is the part of the application to handle user interaction..

The system uses web technology based on Java language. The frontend HTML / CSS / JS there, AJAX/JQuery do web pages, servlet / JSP to achieve the use of interaction between the browser and the web server, the system framework is constructed using Struts2 framework, and the Oracle database as the end of the data storage, the students end to upload the paper - management end delivery - expert side marking the whole network of.

\section{Description of the System Function Module}

The system according to the whole process of the paper review, and from the paper review system has been developed, the overall framework design of the four ports on the management side, expert side, student and tutor end. When the user logs in need through user authentication, to avoid illegal users enter the system.

Management End. Set the system administrator, the school / the office of the office of the degree, two college secretary and other user roles. Including system management, student management, tutor management, expert management, paper management five major functional modules.

System Management. Including organization, role management, user management, review date, data dictionary, system log 6 sub modules. According to the different functions of each role in the paper review process, the system administrator can reasonably assign the authority of the user role in the management side.

Student Management. Including student information management, student information into and out of the list of 3 sub trial. The system administrator assigns the students the corresponding authority, is responsible for the introduction, the revision, the deletion student's related information.

Tutor Management. Including tutor information management, tutor information into 2 sub modules. The system administrator assign the corresponding authority, responsible for the import, modify, delete the relevant information of the tutor, but also can be set as the expert advisor.

Pert Management. Including expert information management, expert information into 2 sub module; system administrator assigned to review the appropriate authority of experts, responsible for import, modify, delete the relevant information of experts. Extensive collection of relevant information, the establishment of expert resource library is the key to ensure the quality of the paper review.

Paper Management. Contains the library management, query the delivery, review, SMS send messages, emails, text messages, the operation log 12 sub module is the assessment of the main module. This system designed the operation log module, the administrator can view each role of the paper's operation process, can be a good track of the review process, provide the control of the paper review process.

Student End. Including information maintenance, paper management two major functional modules. Students to the administrator of the distribution of access to the system, complete the personal information input and maintenance, and complete the paper upload.

Expert End. Including information maintenance, paper management two major functional modules. To enter the system, the authority of the administrator to complete the personal information entry and maintenance, and complete the paper review.

Mentor End. Including information maintenance, paper management two major functional modules. Instructors to the administrator of the distribution of access to the system, complete personal information entry and maintenance, and can be viewed as a guide for students to upload the paper and review the situation. At the same time, the tutor can also be used as experts, complete the review.

\section{System Review Function}

Review system for the delivery of the process is the students to upload the paper, by the management end of the delivery of the end to experts, expert login system to be delivered the 
marking, after the completion of the grading submissions print and send them back to the sending agency, management terminal can also in the expert marking on the management review of the relationship between view grading submissions.

Implementation of the Thesis Submission. The system administrator in accordance with the school rules, such as the name of encoding login account and password assigned to students. After entering the system, the students fill in the basic information such as personal information, tutor information, paper information, etc.. After the completion of the above operation, the students, college secretary, academic degree office staff can be timely access to papers uploaded and converted.

Realization of the Thesis Distribution. Three kinds of delivery mode of the system design. First, the establishment of a direct relationship with experts. This model requires the system administrator early after a lot of work, the establishment of expert database and delivery operation personnel according to the involved in professional and research directions and information in the platform in the query and the corresponding experts, establish the evaluation relation. Two is the review of the paper through the system operation randomly generated assessment number and password, the accreditation number that is the expert login account, distributed to the assessment expert. Three is the review paper sent to the other party school degree work office, according to the specific management of the type of paper, involved in the professional sent to the relevant experts.

The Realization of the Thesis Review. Experts get login account and password, enter the assessment system, can be online or download to see the need to review the paper. And fill out the online review submissions. After the completion of all the links, click Submit. At this time the system administrator has been in platform system view to marking, through the audit, students can also view the marking for the first time, so that timely modification. At the same time to ensure the efficiency of the review, the system also designed two functions, one is the development of the message and the phone to remind the function, through the mail, the phone prompts experts to review. Experts such as a result of various reasons can not be reviewed, but also through this channel feedback, so that we timely replacement assessment expert. Two is the assessment of the assessment cycle, that is, within the specified time, the experts can review the operation, once the deadline, you can no longer see the review paper.

Realization of the Thesis Feedback. After the completion of the expert review, the system can be summarized as a summary of the overall assessment of the situation and the assessment level, the formation of statistical reports, so that the degree of staff, to guide the analysis and evaluation of teachers finishing.

In the process of the actual operation evaluation system, we found that the system still has some problems and shortcomings, such as the compatibility of the system, the transfer of the frame of the paper, the function of the operation is still need to be improved. One is to improve the compatibility of the system, as far as possible to ensure that different network operating environment, can ensure the normal review papers. Second is to further improve the system function, ensure the upload, conversion, marking process, accurate, and further to simplify the process of operation, strengthen the function of the system, easy to use.

\section{The Implementation of Network Evaluation of Thesis}

Develop Technical Standards. The standardization of software documents and procedures is helpful to improve the consistency, integrity and quality of the software, so it is easy to save, exchange and reuse. The system according to the part of the software development standards, the use of uniform standards and standards. See table below for details. 
Table 1 International Standards for Software Development

\begin{tabular}{|c|c|}
\hline Specification for computer software development & GB8566-88 \\
\hline $\begin{array}{c}\text { Guide for the preparation of computer software product development } \\
\text { documentation }\end{array}$ & GB8567-88 \\
\hline Guidelines for the preparation of computer software requirements & GB9385-88 \\
\hline Specification for computer software test documentation & GB9386-88 \\
\hline Information processing program structure and its representation & GB/T13502-92 \\
\hline Computer software unit test & GB/T15532-95 \\
\hline Software maintenance guide & GB/T14079-93 \\
\hline Guidelines for the preparation of computer software requirements & GB/T9385-88 \\
\hline Guide for the preparation of computer software test documentation & GB/T9386-88 \\
\hline Specification for computer software quality assurance program & GB/T12504-90 \\
\hline Computer software reliability and maintainability management & GB/T14394-93 \\
\hline Software product evaluation quality characteristics and guidelines for its use & GB/T16260-96 \\
\hline
\end{tabular}

Establish Expert Resource Database. Establish a rich pool of experts in the network evaluation system is the key to guarantee the quality of the review, in our school system to establish and perfect the expert resource base, mainly take the school teacher and school teacher way of selecting a combination of. On the one hand, to encourage the academic level of outstanding teachers as accreditation experts, will be included in the expert resource library, the Graduate School of the various types of expert information input system. On the other hand, graduate school according to the years of brother institutions experts to review the situation and to outside experts were further screening, the high academic level, business ability, marking the work of a conscientious and responsible experts into the expert resource pool, and enrich the expert resources.

Technical Training. After the review system research and development, in order to make the school teachers and students understand the operation process of the system as soon as possible, familiar with the operation evaluation system, the school coordination technical personnel organized the relevant technical training. One is for the training of management personnel, including specific operating system staff and the school of health secretary, mainly for system organizational structure, role permission assignment, operation each link, add and delete information content to explain in detail; second is for graduate students and teacher training, including how to upload papers, view the and marking submissions, modify personal information and other content is described in detail.

\section{The Operation Guarantee of Network Evaluation System}

Equipment Support. To establish a computer hardware equipment support system, to ensure the normal operation of the network evaluation system of graduate degree thesis. One is the system of computers must use the uninterruptible power supply (UPS), to avoid unexpected power outages cause data loss, affect the work; the second is the system of computer are not free to delete the system software and program software of computer hard disk file.

Personnel Security. In addition to the system administrator, also equipped with professional and technical personnel to maintain the safe operation of the system. System administrators responsible for students, instructors, experts and other information input, and the maintenance of academic institutions and other information, such as the running process of the system problems, the technology needed to solve, by the administrator is responsible for contact technical personnel. Technical personnel responsible for technical issues, provide specific solutions to ensure the normal operation of the system.

Institutional Guarantee. In order to ensure the stable operation of the system, should be strictly in accordance with the review process; hardware needs to be implemented in accordance with the requirements of the equipment to ensure that the problem of computer equipment to prevent the normal operation of the system. 


\section{Reference}

[1] Guo Wenrong. Design and implementation of the evaluation system of University thesis [J]. computer and network, 2014 (12): 59-61

[2] Yu Wenjie. The design and implementation of the evaluation system of student's degree thesis of Art Institute [D]. Yunnan University, University of Electronic Science and technology, 2012

[3] $\mathrm{Hu}$ Jingpo, Li gatehouse. Computer design and implementation of [J]. Dissertation Evaluation Information Platform Based on Web and digital engineering, 2009, (4): 29-32.

[4] Zhou Jianhui, Song Chuping. Design and implementation of ASP.NET based paper review system in Nantong [J]. Journal of Chinese University, 2008, (4): 80-82.

[5] Xu Jing, Li Zhiqiang, Jie Shen. The realization of expert online assessment system [J]. Journal of Yangzhou University, 2004, (4): 63-64. 